Check for updates

Cite this: RSC Adv., 2017, 7, 55571

\title{
3D inkjet printing of star block copolymer hydrogels cross-linked using various metallic ions $\uparrow$
}

\begin{abstract}
Y. Nakagawa, ${ }^{a}$ S. Ohta, ${ }^{\text {b M. Nakamura }}{ }^{\text {c }}$ and T. Ito (D) *ab
Novel precursor polymers for three-dimensional (3D) inkjet printing of hydrogels are required for various biomedical applications. We have, for the first time, investigated 3D inkjet printing of ionically crosslinked star block copolymer hydrogels, for which the precursor polymer has a dendritic polyester core, a poly(oligo(ethylene glycol) methyl ether acrylate) inner layer, and a poly(acrylic acid) outer layer. The star block copolymer solution ( 8.0 wt\%) showed a viscosity of $7-8 \mathrm{mPa}$, which is suitable for inkjet printing. This solution formed a homogeneous hydrogel upon the addition of metallic ions, such as the zinc, copper(II), aluminum, and ferric ion. The elasticity of the resulting hydrogels was dependent on the ion species. The rapid sol-gel transition induced by the metallic ions enabled 3D inkjet printing of the star block copolymer hydrogels, through ejection of the star block copolymer solution and subsequent ionic cross-linking to achieve layer-by-layer deposition of the gelled droplets.
\end{abstract}

Received 18th October 2017 Accepted 2nd December 2017

DOI: 10.1039/c7ra11509a

rsc.li/rsc-advances combination with other materials) ${ }^{5}$ and fibrin, ${ }^{17,18}$ have been reported as useful for 3D inkjet printing. Since the suitable viscosity range for the ink-jetting process is very low, usually $3.5-12 \mathrm{mPa} \mathrm{s},{ }^{15}$ the $3 \mathrm{D}$ inkjet printing of hydrogels has been achieved mainly through an in-process cross-linking approach, ${ }^{19}$ where the ejection of precursor solution and subsequent cross-linking of the droplets are repeatedly conducted to achieve layer-by-layer deposition of the hydrogel. In this process, extremely fast gelation, as well as low viscosity of the precursor solution, is required to avoid the droplet spreading upon impact onto the already-gelled surface. These strict requirements have precluded use of hydrogels other than alginate or fibrin. Therefore, further investigation of 3D-inkjetprintable hydrogels that allow a facile molecular design, as well as large-scale synthesis in an affordable manner, is required.

We have previously reported a star block copolymer that can form a homogeneous hydrogel without polymer precipitation through mixing with calcium ion $\left(\mathrm{Ca}^{2+}\right)^{20}$ or ferric ion $\left(\mathrm{Fe}^{3+}\right){ }^{21}$ This star block copolymer has a dendritic polyester (DPE) core, a poly(oligo(ethylene glycol) methyl ether acrylate) (polyOEGA) inner layer, and a poly(acrylic acid) (PAA) outer layer (Fig. 1a). In the current study, 3D inkjet printing of this synthetic, star block copolymer hydrogel was investigated. Since the star block copolymer solution shows fast gelation upon the addition of metallic ions, this material can be used in 3D inkjet printing

\footnotetext{
${ }^{a}$ Department of Chemical System Engineering, The University of Tokyo, 7-3-1 Hongo, Bunkyo-ku, Tokyo 113-8656, Japan

${ }^{b}$ Center for Disease Biology and Integrative Medicine, The University of Tokyo, 7-3-1 Hongo, Bunkyo-ku, Tokyo 113-8655, Japan.E-mail: taichi@m.u-tokyo.ac.jp

${ }^{c}$ Graduate School of Science and Engineering, University of Toyama, 3190 Gofuku, Toyama 930-8555, Japan

$\dagger$ Electronic supplementary information (ESI) available: Additional pictures of the hydrogels, loss tangent data of the hydrogels, and gelation kinetics. See DOI: 10.1039/c7ra11509a
}

through an in-process cross-linking approach (Fig. 1b).

\section{Experimental}

\section{Materials}

Dendritic polyester (DPE; Boltorn ${ }^{\circledR}$ H20) was kindly provided by Perstorp Japan Co., Ltd. (Tokyo, Japan). Oligo(ethylene glycol) 


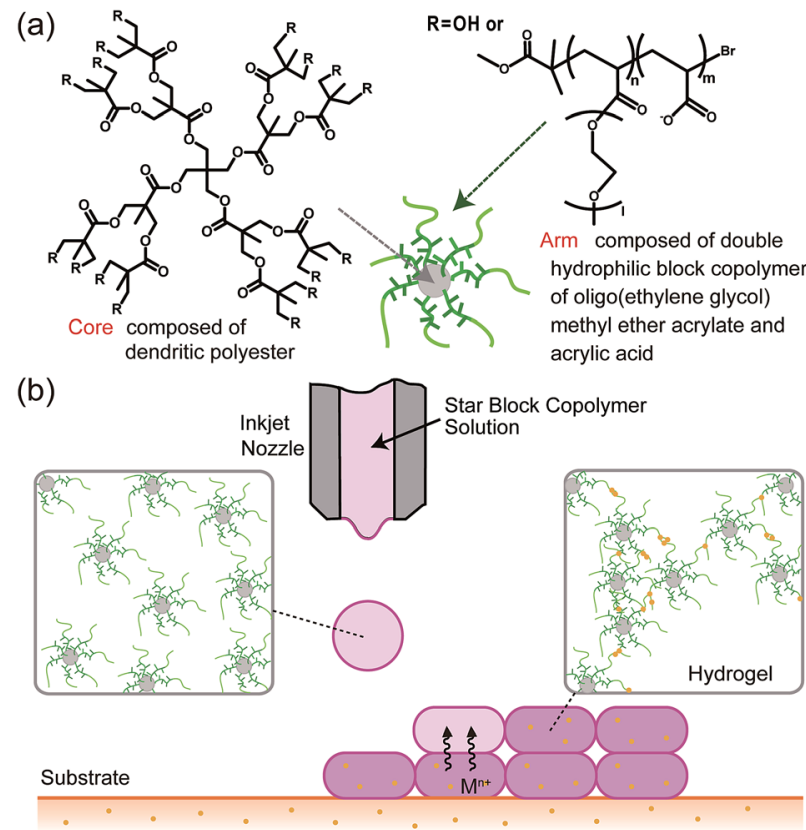

Fig. 1 (a) Schematic illustration of the star block copolymer used as a precursor polymer. (b) Schematic illustration of 3D inkjet printing of the star block copolymer hydrogel cross-linked using metallic ions through an in-process cross-linking approach. The precursor polymer solution was ejected from an inkjet nozzle onto the agarose gel substrate containing a metallic ion $\mathrm{M}^{n+}$ (yellow dots). The ejected droplets show gelation upon impact onto the substrate as a result of upward-diffusion of the metallic ion, thus enabling layer-by-layer deposition of the gelled droplets.

methyl ether acrylate (OEGA; AM-90G) was kindly gifted by ShinNakamura Chemical Co., Ltd. (Wakayama, Japan). Zinc chloride $\left(\mathrm{ZnCl}_{2}\right)$, copper(II) chloride $\left(\mathrm{CuCl}_{2}\right)$, aluminum chloride hexahydrate $\left(\mathrm{AlCl}_{3} \cdot 6 \mathrm{H}_{2} \mathrm{O}\right)$, iron(III) chloride hexahydrate $\left(\mathrm{FeCl}_{3} \cdot 6 \mathrm{H}_{2} \mathrm{O}\right), t$-butyl acrylate $(t \mathrm{BA})$, and agarose were purchased from Wako Pure Chemical Industries, Ltd. (Osaka, Japan). Chelate resin (DIAION CR20) was kindly provided by Mitsubishi Chemical Corporation (Tokyo, Japan). Ethylenediamine- $N, N^{\prime}$-disuccinic acid trihydrate (EDDS) was kindly provided by Chubu Chelest Co. Ltd. (Osaka, Japan). Dialysis membranes (Spectra/Por, MWCO $=6-8 \mathrm{kDa}$ ) were purchased from Spectrum Laboratories Inc. (Rancho Dominguez, CA, USA). Ultrafiltration membranes (NTU-2120, MWCO $=20 \mathrm{kDa}$ ) were kindly provided by Nitto Denko Corporation (Osaka, Japan).

\section{Synthesis of the star block copolymer (DPE- $\boldsymbol{g}$-OEGA- $\boldsymbol{b}$-AA)}

The star block copolymer (DPE- $g$-OEGA- $b$-AA) was synthesized in accordance with the procedure described in our previous publication. ${ }^{20}$ Briefly, the terminal hydroxyl groups of DPE were initially converted into 2-bromoisobutyryl groups to give the DPE-based macroinitiator (DPE-Br). ${ }^{22}$ Using the DPE-Br as an initiator, the star arms were then polymerized through sequential atom transfer radical polymerization (ATRP) ${ }^{23}$ reactions of OEGA and $t$ BA. The first ATRP step yielded a star polymer with a DPE core and polyOEGA arms (DPE-g-OEGA), and the second ATRP step yielded a star block copolymer with a DPE core, a polyOEGA inner layer, and a polytBA outer layer (DPE- $g$-OEGA- $b$ - $t \mathrm{BA})$. The resulting polymer was subject to an acid hydrolysis reaction to allow for the deprotection of the $t$-butyl groups. The deprotected polymer was then neutralized to allow it to be solubilized in distilled water to give the final product (DPE- $g$-OEGA- $b$-AA). The copper catalysts used for the ATRP reactions were removed from the star polymers as follows. After each ATRP reaction, the reaction mixture was diluted using excess acetone and was then mixed with the chelating resin. The resulting mixture was agitated overnight to allow for the removal of the copper catalysts. Crude polymer (DPE- $g$ OEGA or DPE- $g$-OEGA- $b$ - $t \mathrm{BA}$ ) was then obtained through removal of the chelating resin and subsequent evaporation. For further removal of the copper catalysts, the obtained DPE- $g$ OEGA or DPE- $g$-OEGA- $b$-AA (after deprotection of the $t$-butyl groups) was dissolved in the EDDS aqueous solution, and the resulting polymer solution was dialyzed against distilled water. Note that the dialyzed DPE- $g$-OEGA solution was further concentrated using ultrafiltration. For more details on the removal of copper catalysts, see our previous report. ${ }^{20}$ Synthesis of the DPE- $g$-OEGA- $b$-AA polymer was confirmed through ${ }^{1} \mathrm{H}$ NMR and FT-IR measurements as discussed in our previous report. ${ }^{20}$

\section{Viscosity measurements}

Viscosity measurements were conducted using a DV-II + Pro cone-type viscometer (Brookfield AMETEK, Inc., Middleboro, MA, USA) at $25{ }^{\circ} \mathrm{C}$ using a cone spindle (CP52; diameter $=$ $24 \mathrm{~mm}$, cone angle $=3$ degrees). The star block copolymer was dissolved in saline at a concentration of $5-20 \mathrm{wt} \%$, and the solution viscosity was measured with varying shear rate.

\section{Evaluation of gelation ability}

The star block copolymer was dissolved in saline (16 wt\%). The polymer solutions ( $20 \mu \mathrm{L}$ each) were then treated using $20 \mu \mathrm{L}$ of $\mathrm{ZnCl}_{2}, \mathrm{CuCl}_{2}, \mathrm{AlCl}_{3}$, or $\mathrm{FeCl}_{3}$ at varying concentrations in saline, and the resulting mixtures were stirred gently at room temperature. After $5 \mathrm{~s}$, the mixtures were observed to determine whether gelation had occurred or not.

\section{Dynamic viscoelasticity measurements}

Dynamic viscoelasticity measurements were conducted using a rheometer (MCR301; Anton Paar, Graz, Austria) with a parallel-plate geometry (PP25; diameter $=25 \mathrm{~mm}$, gap $=$ $1.0 \mathrm{~mm}$ ). For these measurements, $8.0 \mathrm{wt} \%$ star block copolymer hydrogels cross-linked using $\mathrm{Zn}^{2+}, \mathrm{Cu}^{2+}, \mathrm{Al}^{3+}$, or $\mathrm{Fe}^{3+}$ were prepared as follows. The star block copolymer solution in saline $(8.0 \mathrm{wt} \%)$ was cast into silicone molds (diameter $=25 \mathrm{~mm}$, depth $=1.0 \mathrm{~mm}$ ), which were further sealed using dialysis membranes. Subsequently, the silicone molds containing the polymer solution were immersed in $160 \mathrm{mM} \mathrm{ZnCl}_{2}, \mathrm{CuCl}_{2}$, $\mathrm{AlCl}_{3}$, or $\mathrm{FeCl}_{3}$ solution in saline $(300 \mathrm{~mL})$ overnight, to obtain homogeneous hydrogel disks. The frequency dependencies of the storage modulus $\left(G^{\prime}\right)$, loss modulus $\left(G^{\prime \prime}\right)$ and loss tangent $(\tan \delta)$ of all of the hydrogel samples were measured at a strain 
value of 5\%. Dynamic strain sweep tests were performed for all the samples from 0.1 to $10 \%$ at $1 \mathrm{~Hz}$ to confirm that this strain was within the linear-viscoelastic regime. In order to evaluate gelation time of the above-mentioned samples, time dependence of $G^{\prime}$ and $G^{\prime \prime}$ of the mixture of $8.0 \mathrm{wt} \%$ star block copolymer and $160 \mathrm{mM}$ metallic ion $(500 \mu \mathrm{L})$ was further measured. The strain value and the frequency were fixed at $5 \%$ and $1 \mathrm{~Hz}$, respectively.

\section{D inkjet printing of the star block copolymer hydrogels}

The 3D printing of the star block copolymer hydrogels was conducted using a custom-made inkjet printer with a Fuji piezoelectric print head (Fuji Electric Systems, Tokyo, Japan), as reported previously. ${ }^{24}$ The print head has semicircular nozzles with a diameter of $\sim 70 \mu \mathrm{m}$. The star block copolymer solution in saline $(8.0 \mathrm{wt} \%)$ was syringe filtered $(0.2 \mu \mathrm{m})$ before printing. For visualization of the 3D-printed hydrogels, red dye $\left(35 \mu \mathrm{g} \mathrm{mL}{ }^{-1}\right.$; red food dye, Kyoritsu Shokuhin Co. Ltd., Tokyo, Japan) was added to the star block copolymer solution. Agarose gels containing metallic ions were prepared as substrates for the 3D gel printing. The agarose gels were prepared by casting boiled agarose solution ( $5 \mathrm{wt} \%$ ) into plastic molds with a diameter of $35 \mathrm{~mm}$ and a depth of $10 \mathrm{~mm}$, and allowing subsequent cooling in a refrigerator. The obtained agarose gels were then immersed in saline containing $160 \mathrm{mM} \mathrm{ZnCl}, \mathrm{CuCl}_{2}, \mathrm{AlCl}_{3}$, or $\mathrm{FeCl}_{3}$ overnight.

The honey-comb-shaped star block copolymer hydrogels were $3 \mathrm{D}$-printed at room temperature using layer-by-layer printing of 20 images onto the gel substrate mentioned above using the star block copolymer solution as an ink. According to the previous report published by our group ${ }^{24}$ process parameters were set as follows. Distance from the printer to the substrate was fixed at $4 \mathrm{~mm}$. Head-speed and dot-pitch were $32000 \mu \mathrm{m} \mathrm{s}^{-1}$ and $48 \mu \mathrm{m}$, respectively. The 3D-printed hydrogels were removed from the substrate through the gentle addition of saline onto the substrate to allow the hydrogels to float.

\section{Results and discussion}

The star block copolymer was synthesized through a core-first approach using atom transfer radical polymerization (ATRP), ${ }^{23}$ in accordance with a previous report. ${ }^{20}$ The terminal hydroxyl groups of DPE were transformed to 2-bromoisobutyryl groups, which function as ATRP initiators. The star arms were then polymerized from the DPE core by sequential ATRP of OEGA and $t$-butyl acrylate to obtain a star block copolymer. The $t$-butyl groups of the star block copolymer then underwent deprotection using trifluoroacetic acid to obtain the final product. As reported previously, ${ }^{20}$ the average number of arms was determined to be approximately 9 from the ${ }^{1} \mathrm{H}$ NMR spectra, and the degree of polymerization of the polyOEGA and PAA block was calculated to be 18 and 37 , respectively, from the ${ }^{1} \mathrm{H}$ NMR spectra.

With a view to using the star block copolymer solution as a precursor ink, the printability of the material through an inkjet nozzle was examined. We measured the solution viscosity

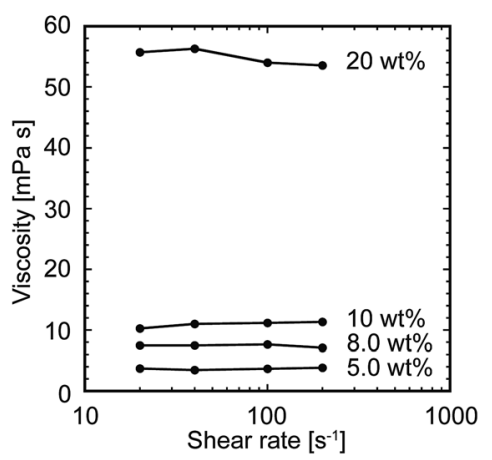

Fig. 2 Viscosity of the star block copolymer solution at different concentrations as a function of shear rate. Saline was used as a solvent.

of the star block copolymer at varying shear rate (Fig. 2), which is an important measurement to determine printability. The solution viscosity depended on the polymer concentration and varied from 3-4 mPa s at $5 \mathrm{wt} \%$ to $54-56 \mathrm{mPa}$ s at $20 \mathrm{wt} \%$. From this result, considering that the viscosity range required for inkjet-printable materials is reported to be $3.5-12 \mathrm{mPa} \mathrm{s},{ }^{15}$ a star block copolymer solution at a concentration below $10 \mathrm{wt} \%$ would be preferable for inkjet-printing. Therefore, for the following experiments, we fixed the polymer concentration at $8.0 \mathrm{wt} \%$, where the solution viscosity was $7-8 \mathrm{mPa}$.

Compared with conventional precursor polymers for the 3D inkjet printing of hydrogels, such as alginate, the star block copolymer showed a much lower solution viscosity. The viscosity of the $5.0 \mathrm{wt} \%$ star block copolymer solution was 3-4 mPa s, whereas that of a $5.0 \mathrm{wt} \%$ alginate solution was $500-1500 \mathrm{mPa}$ s (data not shown). Alginate shows high solution viscosity because of the rigidity of the polymer chain, especially at GG blocks, and electrostatic repulsion arising from the carboxylic moieties. As a result of this high solution viscosity, the concentration of an inkjetprintable alginate solution is reported to be at most $\sim 2 \mathrm{wt} \%{ }^{25}$ On the other hand, the solution viscosity of the star block copolymer was within the viscosity range of inkjet-printable materials $(3.5-12 \mathrm{mPa} \mathrm{s})^{15}$ even at $10 \mathrm{wt} \%$. The star-shaped structure would contribute to the decrease of the solution viscosity; star-shaped polymers have been reported to show lower solution viscosity compared with linear polymers with the same molecular weights. ${ }^{26}$ This low solution viscosity of the star block copolymer is one of the advantages when considering its application as an ink material.

We then evaluated the gelation ability of the star block copolymer solution through cross-linking using several metallic ions, such as zinc $\left(\mathrm{Zn}^{2+}\right)$, copper(II) $\left(\mathrm{Cu}^{2+}\right)$, aluminum $\left(\mathrm{Al}^{3+}\right)$, and $\mathrm{Fe}^{3+}$. While use of $\mathrm{Ca}^{2+}$ or $\mathrm{Fe}^{3+}$ as a cross-linker to generate the star block copolymer hydrogel has been previously reported by our group, ${ }^{\mathbf{2 0 , 2 1}}$ that of other metallic ions has not been investigated. We simply added $20 \mu \mathrm{L}$ of the metallic ion solution in saline to $20 \mu \mathrm{L}$ of the $16 \mathrm{wt} \%$ star block copolymer solution in saline (Fig. S1 $\dagger$ ). The star block copolymer solution showed gelation upon addition of $\mathrm{Zn}^{2+}, \mathrm{Cu}^{2+}, \mathrm{Al}^{3+}$, or $\mathrm{Fe}^{3+}$ aqueous solution at a concentration above a certain threshold. Interestingly, the amount of the metallic ion required to induce gelation was different depending on the ion species in the order of $\mathrm{Zn}^{2+}>\mathrm{Cu}^{2+}>\mathrm{Al}^{3+} \sim \mathrm{Fe}^{3+}$. 
We further evaluated the viscoelasticity of the obtained hydrogels. We fixed the ratio of the concentration of the metallic ion to that of the carboxylate $\left(\left[\mathrm{M}^{n+}\right] /\left[\mathrm{COO}^{-}\right]\right)$at 1.0. We measured the frequency dependencies of the storage modulus $\left(G^{\prime}\right)$ and loss modulus $\left(G^{\prime \prime}\right)$ of the hydrogels using a rheometer (Fig. 3). The measured $G^{\prime}$ and $G^{\prime \prime}$ of the star block copolymer hydrogels cross-linked with $\mathrm{Zn}^{2+}, \mathrm{Cu}^{2+}, \mathrm{Al}^{3+}$, or $\mathrm{Fe}^{3+}$ were frequency-independent, and the $G^{\prime}$ was larger than $G^{\prime \prime}$. These results indicate that all the obtained hydrogels behaved as viscoelastic solids, regardless of the ion species. In addition, the hydrogels showed a different viscoelasticity depending on the ion species used. The loss tangent $(\tan \delta)$, which is a ratio of $G^{\prime \prime}$ to $G^{\prime}$, of the hydrogels cross-linked using $\mathrm{Zn}^{2+}, \mathrm{Cu}^{2+}, \mathrm{Al}^{3+}$, or $\mathrm{Fe}^{3+}$ was $0.554,0.415,0.195$, and 0.112 , respectively (Fig. S2 $\dagger$ ). These results indicate that the elasticity of the hydrogels increases with the ion species, in the following order: $\mathrm{Fe}^{3+}>\mathrm{Al}^{3+}>\mathrm{Cu}^{2+}>$ $\mathrm{Zn}^{2+}$. To evaluate the gelation time, the time dependence of $G^{\prime}$ and $G^{\prime \prime}$ of these materials was also measured at a frequency of $1 \mathrm{~Hz}$ (Fig. S3 $\dagger$ ). As a result, in case of the star block copolymer containing $\mathrm{Cu}^{2+}, \mathrm{Al}^{3+}$, or $\mathrm{Fe}^{3+}, G^{\prime}$ was already larger than $G^{\prime \prime}$ from the beginning of the measurement, indicating that the gelation time was extremely short. In case of the star block copolymer containing $\mathrm{Zn}^{2+}$, on the other hand, $G^{\prime}$ exceeded $G^{\prime \prime} 16$ min after the addition of $\mathrm{Zn}^{2+}$, suggesting that gelation time of this sample was relatively longer compared to the others.

The observed difference in the threshold concentration of the metallic ions for gelation and the viscoelasticity of the resulting hydrogels is considered to result from the variation in (a) $\mathrm{Zn}^{2+}$

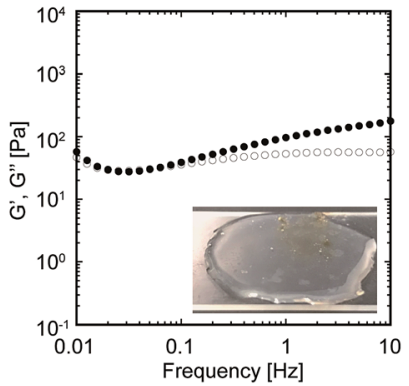

(c) $\mathrm{Al}^{3+}$

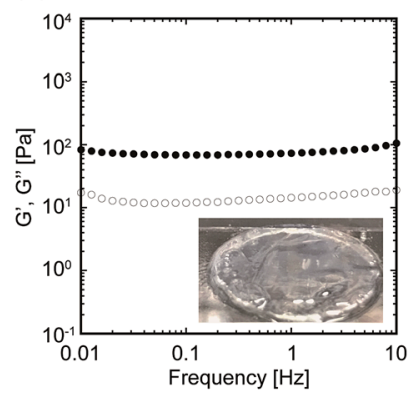

(b) $\mathrm{Cu}^{2+}$

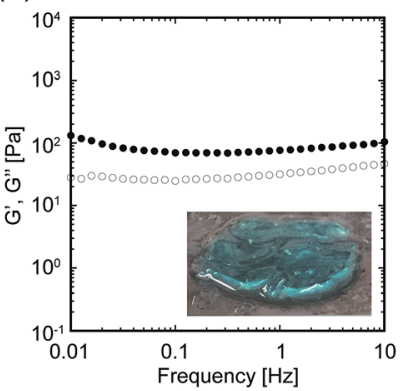

(d) $\mathrm{Fe}^{3+}$

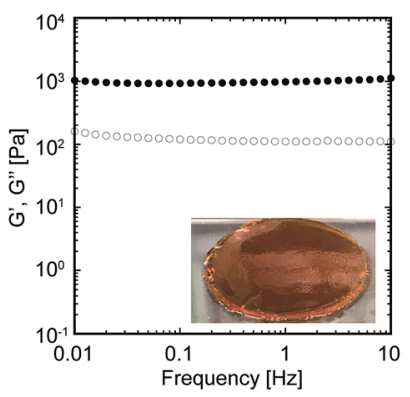

Fig. 3 Frequency dependencies of the storage modulus $G^{\prime}$ (closed circles) and loss modulus $G^{\prime \prime}$ (open circles) of the $8.0 \mathrm{wt} \%$ star block copolymer cross-linked using (a) $\mathrm{Zn}^{2+}$, (b) $\mathrm{Cu}^{2+}$, (c) $\mathrm{Al}^{3+}$, or (d) $\mathrm{Fe}^{3+}$. The ratio of the concentration of the metallic ion to that of the carboxylate $\left(\left[\mathrm{M}^{n+}\right] /\left[\mathrm{COO}^{-}\right]\right)$was fixed at 1.0 . The insets are images of the obtained hydrogels. the stability constant of the metal-carboxylate complex depending on the ion species. Henderson et al. ${ }^{27}$ reported that the life-time and strength of the ionic cross-links are determined mainly by the bond stability of the metal-ligand complex, which has a linear relationship with the thermodynamic equilibrium constant of the complex formation. The lifetime and strength of the cross-links are considered to affect the cross-linking density, which would further determine the gelation threshold and stiffness of the resulting hydrogel.

Finally, we examined 3D inkjet printing of the star block copolymer hydrogels cross-linked using metallic ions. We used a custom-made inkjet printer (Fig. 4a), as reported previously. ${ }^{24}$ The hydrogels were 3D-printed via layer-by-layer printing of 20 images, all of which have the same honey-comb-shaped design. To achieve 3D hydrogel printing through an in-process crosslinking approach, the $8.0 \mathrm{wt} \%$ star block copolymer solution was printed onto the agarose gel substrate containing $\mathrm{Zn}^{2+}$, $\mathrm{Cu}^{2+}, \mathrm{Al}^{3+}$, or $\mathrm{Fe}^{3+}$. Upon impact, the ejected droplets were gelled through the up-ward diffusion of the metallic ion (Fig. 4b). By repeating this process layer by layer, the star block copolymer hydrogel was 3D-printed. After printing, patterned 3D hydrogels cross-linked using $\mathrm{Cu}^{2+}, \mathrm{Al}^{3+}$, or $\mathrm{Fe}^{3+}$ were successfully formed and were transferred from the substrate, while that cross-linked using $\mathrm{Zn}^{2+}$ was just spread on the substrate and was unable to maintain its structure when transferred from the substrate (Fig. 4c). The poor 3D-printability of the hydrogel cross-linked using $\mathrm{Zn}^{2+}$ would be due to the long gelation time as mentioned above. The appearance of the 3D-printed hydrogels was different depending on the ion species used as a crosslinker; the 3D-printed hydrogels cross-linked using $\mathrm{Fe}^{3+}$ or $\mathrm{Al}^{3+}$ showed a clear edge, while that crosslinked using $\mathrm{Cu}^{2+}$ showed a rather blurred edge (Fig. 4d). These results suggest that the printing resolution was different depending on the ion species used.

The printing resolution is dependent on the droplet spreading on the substrate. Generally, the ejected droplet spreads after its impact on a substrate, leading to a lower printing resolution. ${ }^{28}$ In the $3 \mathrm{D}$ hydrogel printing process, droplet spreading is inhibited by gelation. Therefore, the gelation rate predominantly determines the printing resolution. In the above experiments, the gelation rate is considered to be affected by the quantity of metallic ions required to induce gelation; if more ions are required, more time is needed to allow enough ions to diffuse into the droplet. This is the reason that higher resolution was achieved with $\mathrm{Fe}^{3+}$ or $\mathrm{Al}^{3+}$, which requires less ions for gelation.

Further improvement in the printing resolution is also expected to be achieved through optimization of printing process parameters. For example, we previously found that different pattern of driving waveform generated droplets with different shapes, which would result in difference in the printing resolution. Further optimization of distance from the printer to the substrate might ameliorate the printing resolution, considering the fact that drag from air currents in the printing environment affects drop placement accuracy. ${ }^{28}$ In the current system, number of printing layers is also an important parameter to control the printing resolution, because more time is required 
(a)

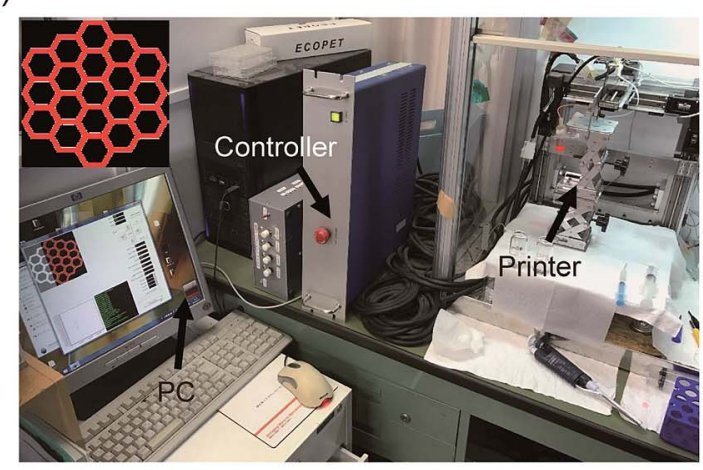

(c)
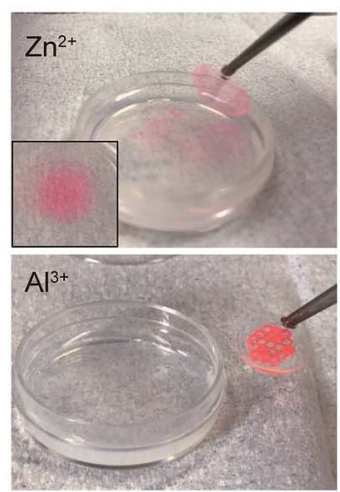

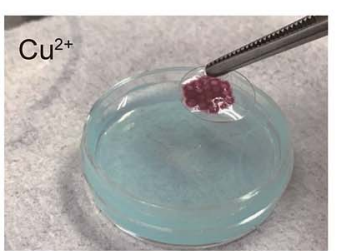

$\mathrm{Fe}^{3+}$

(b)

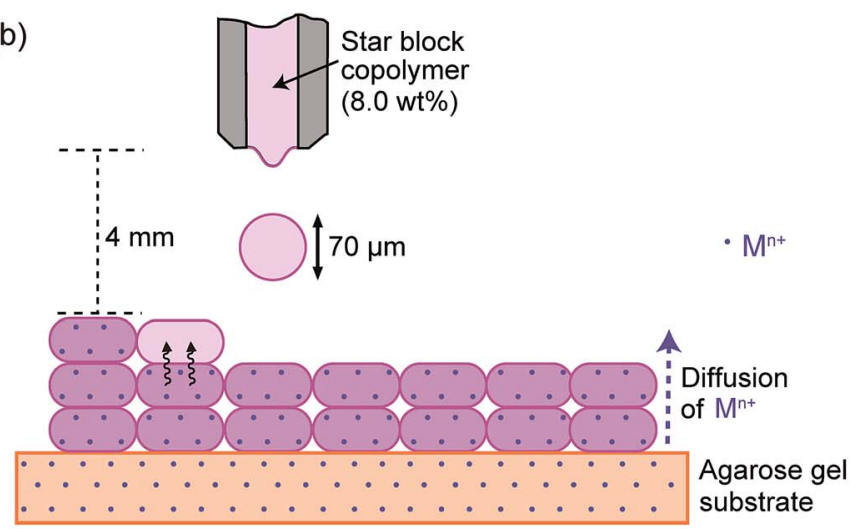

(d)

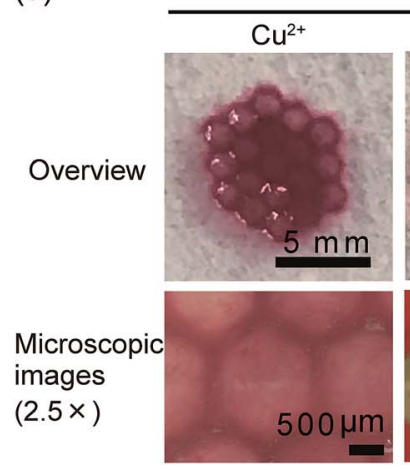

Ion species
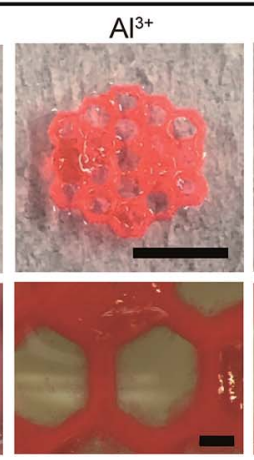

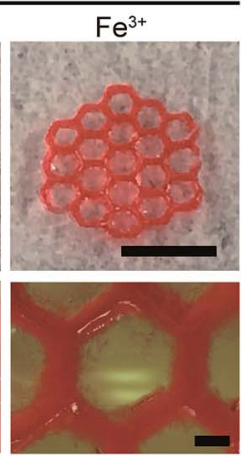

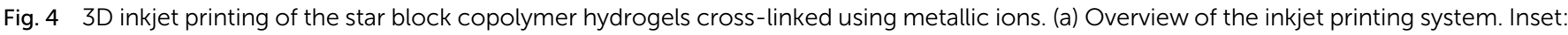

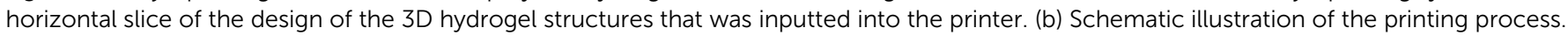

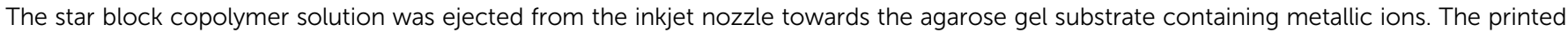

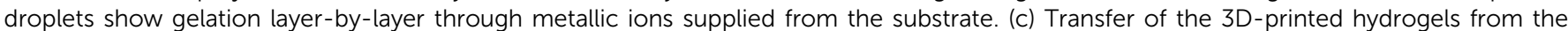

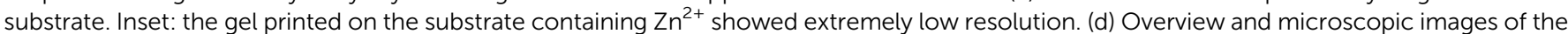

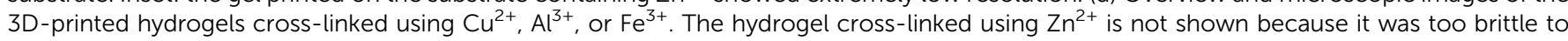
maintain its structure when transferred from the substrate.

to achieve sufficient supply of ions into the droplets deposited onto the higher layers, which would deteriorate the printing resolution of the upper layers. Since substrate surface topography is reported to affect the printing resolution for $2 \mathrm{D}$ printing, ${ }^{15}$ this effect should also be considered when conducting $3 \mathrm{D}$ inkjet printing to improve the printing resolution.

These results represent the successful 3D inkjet printing of ionically cross-linked star block copolymer hydrogels. The 3Dprintable hydrogels must satisfy some criteria; they need to be self-supporting, and must gel extremely rapidly. ${ }^{15}$ In the case of the inkjet systems, in particular, the low droplet viscosity can further accelerate droplet spreading on the substrate, and therefore even faster gelation is required. As a result, 3D-inkjetprintable hydrogels have been less investigated, and alginate gels or gels involving the combination of alginate with other materials have been used in most cases. ${ }^{5}$ Other than alginate gels, a few studies have examined fibrin gels, resulting in rather blurred structures. ${ }^{17,18}$ In the current study, the star block copolymer hydrogels allowed 3D inkjet printing with high resolution comparable to alginate gels. The fast formation of homogeneous hydrogels induced by complexation with free metallic ion is considered to be a key feature, which is achieved by prevention of intra-molecular cross-linking through a strategic molecular design of the precursor polymer. ${ }^{20,21}$
Since the material presented here is composed of a synthetic polymer, it potentially allows engineering of the material by introducing biodegradable star arms or other chelating ligands instead of the carboxylates used in the current polymer, which might decrease the quantity of metallic ions required for gelation to ameliorate the printing resolution. In addition, our material has the potential for scaled-up synthesis in an affordable manner, which is important for future applications. Although a further investigation on the biocompatibility of our materials is required for future biomedical application, our previous research ${ }^{20,21}$ has suggested a high biocompatibility of the star block copolymer hydrogel cross-linked using $\mathrm{Ca}^{2+}$ or $\mathrm{Fe}^{3+}$, as well as low cytotoxicity of the precursor polymer itself. The fast gelation rate of our material would also enable 3D inkjet printing of cell-loaded hydrogels in culture media. This system represents a promising platform technology to expand the design flexibility of 3D-inkjet-printable hydrogels, which have potential for a variety of applications.

\section{Conclusions}

Utilizing a star block copolymer with a DPE core, a polyOEGA inner layer, and a PAA outer layer as a precursor ink, we have, for the first time, demonstrated 3D inkjet printing of synthetic, 
star block copolymer hydrogels through the in-process crosslinking approach. The star block copolymer solution (8.0 wt\%) showed a viscosity of 7-8 $\mathrm{mPa} \mathrm{s}$, which is low enough to be ejected from an inkjet nozzle. In addition, this solution demonstrated gelation upon addition of metallic ions, such as $\mathrm{Zn}^{2+}, \mathrm{Cu}^{2+}, \mathrm{Al}^{3+}$, and $\mathrm{Fe}^{3+}$, and the dynamic viscoelasticity of the resulting hydrogels was dependent on the ion species used as a cross-linker. The rapid gelation using metallic ions enabled the $3 \mathrm{D}$ inkjet printing of the star block copolymer hydrogels, through printing of the precursor solution and subsequent ionic cross-linking of the droplet to form a hydrogel layer-bylayer. The resolution of the printed hydrogel also depended on the ion species, and was highest in the case of $\mathrm{Fe}^{3+}$. The current results pave the way for the flexible design of 3D-inkjetprintable hydrogels.

\section{Conflicts of interest}

There are no conflicts to declare.

\section{Acknowledgements}

We offer our deepest thanks to Perstorp Japan Co., Ltd for supplying DPE, Shin-Nakamura Chemical Co., Ltd for supplying OEGA, Mitsubishi Chemical Corporation for supplying chelate resin, Chubu Chelest Co. Ltd for supplying EDDS and Nitto Denko Corporation for supplying the ultrafiltration membrane. We also thank Professor Tei/Chung and Associate Professor Sakai at The University of Tokyo for providing access to a rheometer. Y. N. appreciates a Research Fellowship from the Japan Society for the Promotion of Science (JSPS). This work was supported by a Grant-in-Aid for JSPS Research Fellow (No. 16J08368).

\section{References}

1 S. H. Huang, P. Liu, A. Mokasdar and L. Hou, Int. J. Adv. Manuf. Technol., 2013, 67, 1191-1203.

2 N. Travitzky, A. Bonet, B. Dermeik, T. Fey, I. Filbert-Demut, L. Schlier, T. Schlordt and P. Greil, Adv. Eng. Mater., 2014, 16, 729-754.

3 W. E. Frazier, J. Mater. Eng. Perform., 2014, 23, 1917-1928.

4 R. D. Goodridge, C. J. Tuck and R. J. M. Hague, Prog. Mater. Sci., 2012, 57, 229-267.

5 J. Malda, J. Visser, F. P. Melchels, T. Jungst, W. E. Hennink, W. J. A. Dhert, J. Groll and D. W. Hutmacher, Adv. Mater., 2013, 25, 5011-5028.

6 S. V. Murphy and A. Atala, Nat. Biotechnol., 2014, 32, 773-785.

7 B. Derby, Science, 2012, 338, 921-926.
8 P. Calvert, Science, 2007, 318, 208-209.

9 T. Q. Huang, X. Qu, J. Liu and S. Chen, Biomed. Microdevices, 2014, 16, 127-132.

10 L. Pan, G. Yu, D. Zhai, H. R. Lee, W. Zhao, N. Liu, H. Wang, B. C.-K. Tee, Y. Shi and Y. Cui, Proc. Natl. Acad. Sci. U. S. A., 2012, 109, 9287-9292.

11 C. Cvetkovic, R. Raman, V. Chan, B. J. Williams, M. Tolish, P. Bajaj, M. S. Sakar, H. H. Asada, M. T. A. Saif and R. Bashir, Proc. Natl. Acad. Sci. U. S. A., 2014, 111, 1012510130.

12 W. Zhu, J. Li, Y. J. Leong, I. Rozen, X. Qu, R. Dong, Z. Wu, W. Gao, P. H. Chung and J. Wang, Adv. Mater., 2015, 27, 4411-4417.

13 V. Chan, K. Park, M. B. Collens, H. Kong, T. A. Saif and R. Bashir, Sci. Rep., 2012, $2,857$.

14 M. Gou, X. Qu, W. Zhu, M. Xiang, J. Yang, K. Zhang, Y. Wei and S. Chen, Nat. Commun., 2014, 5, 3774.

15 T. Jungst, W. Smolan, K. Schacht, T. Scheibel and J. Groll, Chem. Rev., 2015, 116, 1496-1539.

16 A. L. Rutz, K. E. Hyland, A. E. Jakus, W. R. Burghardt and R. N. Shah, Adv. Mater., 2015, 27, 1607-1614.

17 T. Xu, C. A. Gregory, P. Molnar, X. Cui, S. Jalota, S. B. Bhaduri and T. Boland, Biomaterials, 2006, 27, 3580-3588.

18 M. Nakamura, S. Iwanaga, C. Henmi, K. Arai and Y. Nishiyama, Biofabrication, 2010, 2, 014110.

19 J. M. Lee and W. Y. Yeong, Adv. Healthcare Mater., 2016, 5, 2856-2865.

20 Y. Nakagawa, Y. Amano, S. Nakasako, S. Ohta and T. Ito, ACS Biomater. Sci. Eng., 2015, 1, 914-918.

21 Y. Nakagawa, S. Ohta, A. Sugahara, M. Okubo, A. Yamada and T. Ito, Macromolecules, 2017, 50, 5539-5548.

22 G. Kreutzer, C. Ternat, T. Q. Nguyen, C. J. G. Plummer, J. A. E. Manson, V. Castelletto, I. W. Hamley, F. Sun, S. S. Sheiko, A. Herrmann, L. Ouali, H. Sommer, W. Fieber, M. I. Velazco and H. A. Klok, Macromolecules, 2006, 39, 4507-4516.

23 K. Matyjaszewski and J. H. Xia, Chem. Rev., 2001, 101, 29212990.

24 K. Arai, S. Iwanaga, H. Toda, C. Genci, Y. Nishiyama and M. Nakamura, Biofabrication, 2011, 3, 034113.

25 H. Gudupati, M. Dey and I. A. Ozbolat, Biomaterials, 2016, 102, 20-42.

26 J. M. J. Frechet, Science, 1994, 263, 1710-1715.

27 K. J. Henderson, T. C. Zhou, K. J. Otim and K. R. Shull, Macromolecules, 2010, 43, 6193-6201.

28 B. Derby, Annu. Rev. Mater. Res., 2010, 40, 395-414. 